\title{
Disminución de la reserva de flujo coronario en pacientes con insuficiencia cardíaca no isquémica
}

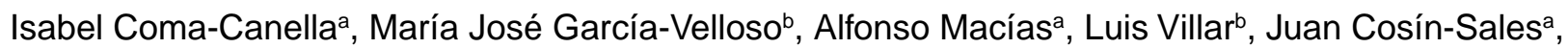
Josep M. Martí-Climent ${ }^{\mathrm{b}}$ yiguel Artaiz ${ }^{\mathrm{a}}$

aDepartamento de Cardiología. Clínica Universitaria de Navarra. Facultad de Medicina. Universidad de Navarra. Pamplona. ' Departamento de Medicina Nuclear. Clínica Universitaria de Navarra

Facultad de Medicina. Universidad de Navarra. Pamplona. España.

Introducción y objetivos. La reserva de flujo coronario (RFC) se reduce no sólo en la cardiopatía isquémica, sino también en otras cardiopatías, con o sin insuficiencia cardíaca. El objetivo del estudio fue comprobar si la gravedad de la insuficiencia cardíaca influye en el deterioro de la RFC.

Métodos. Se estudió a 40 pacientes diagnosticados de cardiopatía no isquémica e insuficiencia cardíaca, en 41 ocasiones distintas. Fueron repartidos en 4 grupos: 1.10 pacientes en grado funcional III-IV; 2. 10 pacientes en grado funcional II sin tratamiento con bloqueadores beta; 3. 11 pacientes en grado funcional II tratados con carvedilol, y 4. 10 pacientes en grado funcional I, que previamente habían tenido insuficiencia cardíaca por disfunción sistólica. El flujo miocárdico (FM) se midió mediante tomografía por emisión de positrones (PET) y N-13 amonio: en condiciones basales y durante la infusión de trifosfato de adenosina (ATP).

Resultados. El FM máximo y la RFC fueron significativamente más altos en el grupo $4(1,95 \pm 0,58$ y 2,40 \pm $0,95 \mathrm{ml} / \mathrm{min} / \mathrm{g})$ que en el grupo $1(1,02 \pm 0,52$ y 1,46 \pm $0,48 \mathrm{ml} / \mathrm{min} / \mathrm{g})$. La RFC tuvo tendencia a ser mayor en los grupos $2(1,73 \pm 0,72)$ y $3(1,89 \pm 0,75)$ que en el grupo 1. No hubo correlación significativa entre la RFC y las siguientes variables: edad, presión arterial sistólica, índice de masa ventricular, índices de volumen y fracción de eyección de ventrículo izquierdo.

Conclusiones. La función microvascular coronaria está alterada en la insuficiencia cardíaca no isquémica, y dicha alteración se relaciona con la situación funcional, cualquiera que sea la cardiopatía subyacente.

Palabras clave: Tomografía por emisión de positrones. PET. Reserva de flujo coronario. Insuficiencia cardíaca. ATP.

\section{Impaired Coronary Flow Reserve in Patients with Non-Ischemic Heart Failure}

Introduction and objectives. Coronary flow reserve (CFR) is impaired not only in ischemic heart disease, but also in cardiac diseases that may or may not course with heart failure. The aim of the present study was to determine if the severity of heart failure can influence CFR impairment.

Methods. Forty patients with non-ischemic heart disease and heart failure were studied 41 times. Four groups were established: 1. 10 patients in functional class III-IV; 2. 10 patients in functional class II not taking beta-blockers; 3. 11 patients in class II treated with carvedilol, and 4. 10 patients in class I. These patients had a history of heart failure and systolic dysfunction. Myocardial blood flow (MBF) was measured with positron emission tomography (PET) and $\mathrm{N}-13$ ammonia at rest ( $r$ ) and during adenosine triphosphate (ATP) infusion.

Results. MBF and CFR were significantly higher in group $4(1.95 \pm 0.58$ and $2.40 \pm 0.95 \mathrm{ml} / \mathrm{min} / \mathrm{g})$ than in group 1 (1.02 \pm 0.52 and $1.46 \pm 0.48 \mathrm{ml} / \mathrm{min} / \mathrm{g})$. CFR tended to be higher in groups $2(1.73 \pm 0.72)$, and $3(1.89 \pm$ $0.75)$ vs group 1 . No significant correlation was found between CFR and the following variables: age, systolic blood pressure, ventricular mass index, ventricular volume indexes, and ejection fraction.

Conclusions. Coronary microvascular function is impaired in non-ischemic heart failure, and the impairment is related to functional class, regardless of the underlying responsible heart disease.

Key words: Positron emission tomography. PET. Coronary flow reserve. Heart failure. ATP.

Full English text available at: www.revespcardiol.org
Este trabajo ha sido financiado por una beca FIS.

Correspondencia: Dra. Isabel Coma-Canella.

Departamento de Cardiología. Clínica Universitaria de Navarra.

Avda. de Pío XII, 36. 31008 Pamplona. España.

Correo electrónico: icoma@unav.es

Recibido el 16 de mayo de 2002.

Aceptado para su publicación el 11 de noviembre de 2002.

\section{INTRODUCCIÓN}

La capacidad dilatadora de la microvasculatura coronaria o reserva de flujo coronario (RFC) está alterada en pacientes con lesiones coronarias significativas ${ }^{1}$. También puede alterarse en presencia de factores de riesgo coronario ${ }^{2,3}$, en la hipertensión $\operatorname{arterial}^{4}$, en la 


\section{ABREVIATURAS}

RFC: reserva de flujo coronario.

FM: flujo miocárdico.

ATP: trifosfato de adenosina.

PET: tomografía por emisión de positrones.

miocardiopatía hipertrófica ${ }^{5}$ o dilatada ${ }^{6,7}$, y en la hipertrofia secundaria a lesiones valvulares ${ }^{8}$.

La RFC se ha encontrado alterada en la insuficiencia cardíaca experimental ${ }^{9}$. La insuficiencia cardíaca se asocia a activación neurohumoral y alteraciones de la circulación periférica ${ }^{10}$. Es posible que el aumento de citocinas y la reducción del flujo influyan en el desarrollo de la disfunción endotelial de estos pacientes ${ }^{10,11}$. La disfunción endotelial puede contribuir al aumento del tono vasomotor periférico durante el ejercicio $^{12}$ y a un control anormal del flujo sanguíneo braquial $^{13}$. Un estudio experimental ${ }^{9}$ muestra que la disfunción endotelial coronaria con reducción de la RFC aparece antes que la insuficiencia cardíaca. Es posible que la vasoconstricción causada por el aumento de endotelina-1 tenga relación con estas alteraciones, ya que el carvedilol mejora la insuficiencia cardíaca a la vez que reduce las concentraciones de esta sustancia $^{14}$.

La RFC puede medirse con diferentes métodos; sin embargo, la tomografía por emisión de positrones (PET) es la única técnica no invasiva que proporciona una cuantificación absoluta del flujo miocárdico global y regional en $\mathrm{ml} / \mathrm{min} / \mathrm{g}^{15}$.

El objetivo del presente estudio ha sido verificar con PET si los pacientes en insuficiencia cardíaca de causa no isquémica tienen alteración funcional de la microvasculatura coronaria, y si esta alteración depende de la gravedad de la insuficiencia cardíaca en el momento del estudio.

\section{MÉTODOS}

Se estudió a 40 pacientes con cardiopatía de origen no isquémico que estuvieron en insuficiencia cardíaca, en clase funcional III-IV de la New York Heart Association en el momento del estudio o previamente. En total se practicaron 41 estudios PET para medir la RFC. El estudio fue aprobado por el comité ético de la institución y todos los pacientes dieron su consentimiento informado. Se establecieron los siguientes grupos de acuerdo con la situación funcional del paciente cuando se hizo el estudio: 1.10 pacientes en clase IIIIV; 2. 10 pacientes en clase II que no recibieron bloqueadores beta; 3.11 pacientes en clase II, tratados con carvedilol, y 4. 10 pacientes que previamente tuvieron insuficiencia cardíaca con fracción de eyección $<0,45$ y en el momento del estudio, además de estar en clase I, tenían una fracción de eyección $\geq 0,45$. Sólo un paciente fue estudiado en dos ocasiones: la primera vez en el grupo 2 y la segunda en el grupo 3 (después de un año en tratamiento con carvedilol). En todos los pacientes se hizo historia clínica, exploración física, electrocardiograma de 12 derivaciones, ecocardiograma, analítica habitual y angiografía coronaria. Sólo se incluyó a pacientes sin lesiones coronarias significativas.

La tabla 1 muestra las principales características basales de cada grupo. La tabla 2 muestra el diagnóstico, la fracción de eyección, el índice de masa y el de volúmenes de ventrículo izquierdo.

Los pacientes de los grupos 2, 3 y 4 habían estado previamente en clase funcional III-IV y mejoraron con fármacos o cirugía (en caso de lesiones valvulares). Los 4 grupos recibieron digital, diuréticos e inhibidores de la enzima convertidora de la angiotensina, solos o combinados. Además de estos fármacos, todos los pacientes del grupo 3 recibieron carvedilol (el único bloqueador beta usado), en dosis que variaron entre 6,25 y $50 \mathrm{mg} /$ día. El tratamiento fue establecido según el criterio del médico responsable de cada paciente, que en general se ajustó a las recomendaciones basadas en la evidencia ${ }^{16}$. Sólo un paciente del grupo 2

TABLA 1. Características basales de los distintos grupos de pacientes

\begin{tabular}{|c|c|c|c|c|c|}
\hline & Grupo 1 & Grupo 2 & Grupo 3 & Grupo 4 & $p$ \\
\hline Número de pacientes & 10 & 10 & 11 & 10 & \\
\hline Edad (años) & $68 \pm 7$ & $58 \pm 11$ & $60 \pm 10$ & $66 \pm 10$ & 0,127 \\
\hline Varones, n (\%) & $7(70)$ & $7(70)$ & $8(72,7)$ & $8(80)$ & 0,573 \\
\hline IMC & $29,1 \pm 5,7$ & $27,9 \pm 3,7$ & $29,2 \pm 2,8$ & $24,5 \pm 3,7$ & 0,064 \\
\hline Tabaquismo, n (\%) & $1(10)$ & $2(20)$ & $4(36,3)$ & $1(10)$ & 0,287 \\
\hline \multicolumn{6}{|l|}{ Hipertensión } \\
\hline arterial, n (\%) & $3(30)$ & $6(60)$ & $7(63,6)$ & $8(80)$ & 0,343 \\
\hline Diabetes mellitus, n (\%) & $2(20)$ & 0 & $2(18,2)$ & $2(20)$ & 0,596 \\
\hline $\mathrm{CT} / \mathrm{HDL}$ & $5,3 \pm 1,7$ & $4,7 \pm 1,1$ & $4,3 \pm 1,2$ & $4,7 \pm 1,5$ & 0,499 \\
\hline
\end{tabular}

CT/HDL: colesterol total/colesterol HDL; IMC: índice de masa corporal (peso en kg/cuadrado de la estatura en m). 
pudo ser tratado (y entonces pasó al grupo 3) con carvedilol. Los restantes pacientes del grupo 2 tenían alguna contraindicación para el tratamiento con bloqueadores beta.

Todos los pacientes se abstuvieron de ingerir cafeína durante al menos las $24 \mathrm{~h}$ previas al estudio PET. Los fumadores dejaron de fumar al menos una semana antes. Por la mañana recibieron el tratamiento habitual y los estudios PET se hicieron por la tarde.

\section{Tomografía por emisión de positrones}

Las imágenes de transmisión y emisión fueron obtenidas con un tomógrafo Siemens Ecat Exact HR+. Este equipo consta de un sistema de 32 anillos de cristales detectores que permiten obtener, con 32 planos directos y 31 cruzados, 63 imágenes transaxiales simultáneas que cubren un campo de $15,5 \mathrm{~cm}$, con una resolución en modo $2 \mathrm{D}$ de $4,5 \mathrm{~mm}$ transaxiales y de 4,5 mm axiales en el centro del campo de visión.

Se cuantificó el flujo miocárdico (FM) en condiciones basales y durante la hiperemia inducida por ATP usando N-13 amonio y adquisición dinámica PET. Primero se adquirió una imagen de transmisión durante 15 min para corregir la atenuación de fotones. Después de la primera inyección intravenosa de N-13 amonio $(9,25 \mathrm{MBq} / \mathrm{kg}$, hasta un máximo de 740 $\mathrm{MBq}$ ), se adquirieron imágenes seriadas en reposo, con una secuencia dinámica de duración variable (12 imágenes $\times 10 \mathrm{~s}, 4 \times 15 \mathrm{~s}, 4 \times 30 \mathrm{~s}, 3 \times 300 \mathrm{~s})$. El protocolo de adquisición de datos PET ha sido descrito por otros autores ${ }^{17}$. Después de la adquisición del estudio basal, se esperó $50 \mathrm{~min}$ para permitir la caída de radiactividad del $\mathrm{N}-13$ amonio $\left(\mathrm{T}_{1 / 2}=9,9 \mathrm{~min}\right)$. Se infundió ATP durante $6 \mathrm{~min}$ en una dosis de 0,160 $\mathrm{mg} / \mathrm{kg} / \mathrm{min}$. Durante la infusión se determinaron continuamente la frecuencia cardíaca, la presión arterial y el electrocardiograma de 12 derivaciones. En el cuarto minuto de la infusión de ATP se realizó la segunda inyección de N-13 amonio. La adquisición de las imágenes de estrés comenzó en el momento de la inyección y se siguió el mismo protocolo que para las imágenes de reposo.

Procesado de imágenes. Las imágenes fueron reconstruidas usando un filtro Hann con una frecuencia de corte de 0,4 , lo que resulta en una resolución efectiva por plano de $7 \mathrm{~mm}$. Las imágenes transaxiales fueron reorientadas en el eje corto y en los ejes largos vertical y horizontal. Los ángulos de ejes largos horizontal y vertical fueron definidos usando las tres últimas imágenes de la secuencia dinámica, y luego se utilizaron para la reorientación de la secuencia de 23 imágenes. Se utilizaron para el análisis cuantitativo seis secciones contiguas del eje corto correspondiente a la parte media del ventrículo izquierdo.

Cuantificación del FM. El FM regional se calculó de acuerdo con un modelo de tres compartimentos ${ }^{16}$, que representa el N-13 amonio vascular y extravascular, y el N-13 atrapado metabólicamente en forma de glutamina, que permite estimar la constante $\mathrm{K}_{1}$ que representa el FM en $\mathrm{ml} / \mathrm{g} / \mathrm{min}$.

El programa informático utilizado para calcular el FM regional fue desarrollado por Muzic et al ${ }^{18}$. Para determinar la función de entrada de radiactividad, se delimitó una región de interés en los dos planos más basales de la cavidad ventricular izquierda en el eje corto. Se definieron 12 regiones de interés por plano en los seis planos en la última imagen de la secuencia dinámica. Se realizó un muestreo de la colección de imágenes dinámicas y se obtuvieron 72 curvas de actividad-tiempo. El FM regional fue analizado en cuatro áreas del ventrículo izquierdo: anterior, septal, inferoposterior y lateral.

\section{Análisis estadístico}

La estadística descriptiva se expresa como media \pm desviación típica. Las medidas cuantitativas se compararon mediante ANOVA, seguido de la prueba de comparaciones múltiples de Tukey. Las diferencias en porcentajes entre grupos se compararon con la prueba exacta (paquete estadístico PEPI, de J.H. Abramson y P.M. Gahlinger, 1993-2000). Para establecer una posible relación entre variables se empleó la correlación de Pearson. Se calculó el coeficiente de variación del FM regional en reposo para evaluar la heterogeneidad espacial de la perfusión miocárdica ${ }^{19}$, determinando en cada sujeto el cociente entre la desviación típica y el valor medio del FM regional en cuatro áreas de miocardio.

\section{RESULTADOS}

\section{Características basales}

No se encontraron diferencias significativas entre los cuatro grupos de pacientes en: edad, sexo, índice de masa corporal, hábito de fumar, diabetes mellitus, hipertensión arterial, o cociente colesterol total/lipoproteínas de alta densidad (tabla 1). Puesto que la RFC tiende a reducirse a partir de los 70 años ${ }^{20}$, se comprobó que 12 de los pacientes eran mayores de 69 años: tres del grupo 1 , dos del 2 , dos del 3 y cinco del 4 (diferencias sin significación estadística). Tampoco se encontraron diferencias significativas en la proporción de los distintos tipos de cardiopatía entre los cuatro grupos (tabla 2). Todas las valvulopatías fueron mitroaórticas reumáticas o degenerativas, con distintos grados de estenosis e insuficiencia. Tres de ellos tuvieron además insuficiencia tricuspídea importante. Sólo un paciente tuvo miocardiopatía restrictiva de causa no filiada. Ocho de los pacientes del grupo 4 estaban diagnosticados de miocardiopatía dilatada (en seis, de origen hipertensivo). Diez pacientes de este grupo, que previamente tuvieron fracción de eyección reducida y 
TABLA 2. Diagnóstico y variables ecocardiográficas en cada grupo

\begin{tabular}{|c|c|c|c|c|c|}
\hline & Grupo 1 & Grupo 2 & Grupo 3 & Grupo 4 & $\mathrm{p}$ \\
\hline Miocardiopatía dilatada, n (\%) & $6(60)$ & $3(30)$ & $8(72,7)$ & $8(80)$ & 0,671 \\
\hline Valvulopatía mitroaórtica, n (\%) & $4(40)$ & $6(60)$ & $3(27,3)$ & $2(20)$ & 0,654 \\
\hline Miocardiopatía restrictiva, n (\%) & 0 & $1(10)$ & 0 & 0 & - \\
\hline Fracción de eyección, \% & $35 \pm 19$ & $43 \pm 15$ & $34 \pm 14$ & $52 \pm 5$ & $0,045^{\star}$ \\
\hline Índice de masa Vl (g/m²) & $149 \pm 48$ & $159 \pm 76$ & $136 \pm 49$ & $128 \pm 28$ & 0,524 \\
\hline Índice de VTD VI (ml/ $\left./ \mathrm{m}^{2}\right)$ & $122 \pm 68$ & $113 \pm 49$ & $125 \pm 45$ & $92 \pm 35$ & 0,508 \\
\hline Índice de VTS VI (ml/m²) & $83 \pm 65$ & $67 \pm 42$ & $81 \pm 42$ & $43 \pm 19$ & 0,186 \\
\hline
\end{tabular}

*Gupo 4 frente a los grupos 1 y 3. VI: ventrículo izquierdo; VTD: volumen telediastólico; VTS: volumen telesistólico.

TABLA 3. Variables hemodinámicas y flujo coronario en condiciones basales y con ATP

\begin{tabular}{|c|c|c|c|c|c|}
\hline & Grupo 1 & Grupo 2 & Grupo 3 & Grupo 4 & $\mathrm{p}$ \\
\hline $\mathrm{PAS}, \mathrm{mmHg}$ & $137 \pm 22$ & $139 \pm 22$ & $137 \pm 15$ & $154 \pm 37$ & 0,391 \\
\hline $\mathrm{FC} \times \mathrm{PAS}$ basal & $10.287 \pm 2.073$ & $9.360 \pm 2.439$ & $10.120 \pm 2.690$ & $9.388 \pm 2.837$ & 0,753 \\
\hline $\mathrm{FC} \times \mathrm{PAS}(\mathrm{ATP})$ & $10.448 \pm 2.867$ & $11.160 \pm 2.020$ & $12.531 \pm 2.987$ & $11.213 \pm 3.957$ & 0,461 \\
\hline $\mathrm{FM}_{\mathrm{b}}, \mathrm{ml} / \mathrm{g} / \mathrm{min}$ & $0,69 \pm 0,25$ & $0,75 \pm 0,24$ & $0,70 \pm 0,14$ & $0,90 \pm 0,35$ & 0,225 \\
\hline $\mathrm{FM}_{\mathrm{b}}$ normalizado & $0,67 \pm 0,16$ & $0,81 \pm 0,19$ & $0,73 \pm 0,22$ & $1,03 \pm 0,53$ & 0,07 \\
\hline $\mathrm{FM}_{\mathrm{ATP}}, \mathrm{ml} / \mathrm{g} / \mathrm{min}$ & $1,02 \pm 0,52$ & $1,40 \pm 0,71$ & $1,37 \pm 0,71$ & $1,95 \pm 0,58$ & $0,03^{\star}$ \\
\hline $\mathrm{RFC}\left(\mathrm{FM}_{\mathrm{ATP}} / \mathrm{FM}_{\mathrm{b}}\right)$ & $1,46 \pm 0,48$ & $1,73 \pm 0,72$ & $1,89 \pm 0,75$ & $2,40 \pm 0,95$ & $0,03^{*}$ \\
\hline Resistencia coronaria basal & $143 \pm 43$ & $127 \pm 35$ & $139 \pm 28$ & $129 \pm 57$ & 0,773 \\
\hline Resistencia coronaria $_{\text {ATP }}$ & $95 \pm 35$ & $89 \pm 47$ & $88 \pm 49$ & $54 \pm 24$ & 0,114 \\
\hline
\end{tabular}

*Gupo 4 frente a grupo 1. ATP: trifosfato de adenosina; FC: frecuencia cardíaca en lat/min; FM: flujo miocárdico; FM RFC: reserva de flujo coronario.

clase funcional III-IV, mejoraron con tratamiento. Al ser estudiados estaban en clase funcional I con fracción de eyección normalizada.

La fracción de eyección fue significativamente más alta en el grupo 4 que en los grupos 1 y 3 . No se observaron diferencias significativas entre los grupos 2 y 4. Ello se debe a que algunos pacientes del grupo 2, con lesiones valvulares, tenían fracción de eyección normal. No se observó diferencia significativa entre grupos en los índices de masa y de volumen de ventrículo izquierdo (tabla 2).

\section{Hallazgos hemodinámicos}

La tabla 3 muestra que no hubo diferencia significativa en la presión arterial sistólica basal entre los cuatro grupos. El doble producto (frecuencia cardíaca $\times$ presión arterial sistólica), indicativo del consumo de oxígeno miocárdico, aumentó de forma leve pero significativa $(\mathrm{p}=0,001)$ con ATP. No hubo diferencias significativas en este parámetro entre los cuatro grupos, ni en reposo ni durante la infusión de ATP.

\section{Flujo miocárdico}

No se observó heterogeneidad espacial significativa $(\mathrm{p}=0,798)$ en el flujo miocárdico basal $\left(\mathrm{FM}_{\mathrm{b}}\right)$ regional entre las cuatro regiones estudiadas: anterior, septal, inferoposterior y lateral. Por este motivo, se analizó únicamente el $\mathrm{FM}_{\mathrm{b}}$ global.

\section{FM, resistencia coronaria y RFC}

La tabla 3 muestra que no hubo diferencia significativa en el $\mathrm{FM}_{\mathrm{b}}$ entre los cuatro grupos. Ya que el FM aumenta cuando se eleva el doble producto ${ }^{21}$, se calculó el $\mathrm{FM}_{\mathrm{b}}$ normalizado (cociente $\mathrm{FM}_{\mathrm{b}} \times 10.000 /$ doble producto). No se encontró diferencia significativa entre los cuatro grupos, si bien se observaron los valores más altos en el grupo 4 y los más bajos en el grupo 1 .

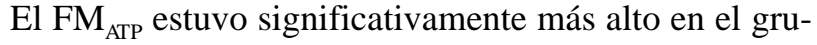
po 4 que en el grupo 1.

Puesto que el ATP desajusta la relación entre FM y trabajo cardíaco, el flujo hiperémico no fue corregido para el doble producto. La RFC (FM ${ }_{\mathrm{ATP}} / \mathrm{FM}_{\mathrm{b}}$ no corregido) fue significativamente más alta en el grupo 4 que en el 1. No se observaron diferencias significativas entre los cuatro grupos en la resistencia coronaria basal (presión arterial media/ $\mathrm{FM}_{\mathrm{b}}$ ) ni en la resistencia coronaria mínima (con ATP), pero hubo una tendencia a menor resistencia coronaria con ATP en el grupo 4 que en los restantes grupos. La figura 1 recoge el $\mathrm{FM}_{\mathrm{b}}$ no corregido, el $\mathrm{FM}_{\text {ATP }}$ y la RFC en los cuatro grupos de pacientes. Comparada con los valores publicados ${ }^{21}$ de $\operatorname{RFC}(3,01 \pm 0,73)$ en voluntarios sanos de $64 \pm 9$ años, la RFC estuvo claramente reducida en los cuatro grupos de pacientes, especialmente en el grupo 1. Aunque la RFC fue ligeramente más alta en los grupos 2 y 3 que en el 1, sólo el grupo 4 tuvo valores significativamente mayores que el 1. El paciente con dos estudios PET tuvo una RFC de 1,63 cuando estaba en el 


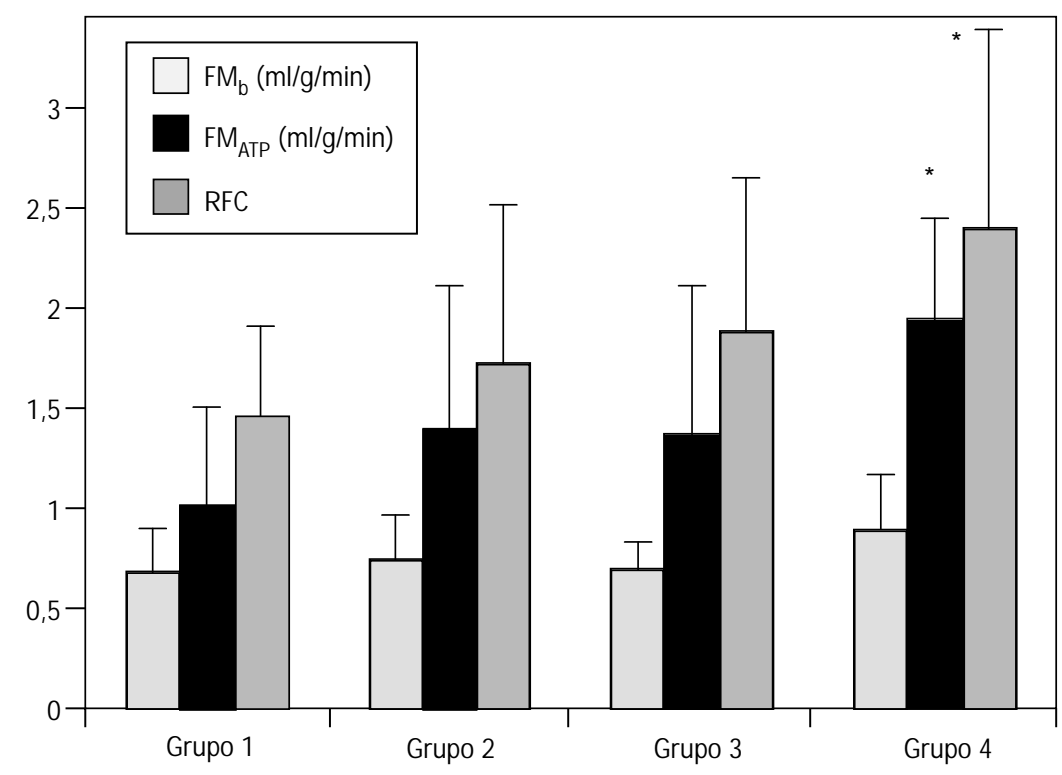

Fig. 1. Representación gráfica del flujo miocárdico (FM) en cada grupo: en condiciones basales $\left(\mathrm{FM}_{\mathrm{b}}\right)$ y con trifosfato de adenosina $\left(\mathrm{FM}_{\mathrm{ATP}}\right)$. La reserva de flujo coronario (RFC) representa el cociente $\mathrm{FM}_{\mathrm{ATP}} / \mathrm{FM}_{\mathrm{b}}$. Sólo el grupo 4 alcanza valores significativamente más altos de $\mathrm{FM}_{\text {ATP }}$ y de RFCque el grupo 1. grupo 2 y de 2,99 cuando estaba en el grupo 3, después de un año en tratamiento con carvedilol. La fracción de eyección fue 0,25 en el primer estudio y 0,35 en el segundo.

No hubo correlación significativa entre RFC y: edad $(\mathrm{r}=0,171 ; \mathrm{p}=0,143)$, presión arterial sistólica $(\mathrm{r}=$ $-0,022 ; \mathrm{p}=0,445)$, fracción de eyección $(\mathrm{r}=0,096$; $\mathrm{p}=0,275)$, índice de masa ventricular izquierda $(\mathrm{r}=$ 0,$047 ; \mathrm{p}=0,388$ ), índice de volumen telediastólico ventricular izquierdo $(r=0,025 ; p=0,439)$, índice de volumen telesistólico ventricular izqierdo $(\mathrm{r}=0,056$; $\mathrm{p}=0,368$ ).

Ninguna de las siguientes covariables modificó significativamente la RFC: edad, índice de masa corporal, fracción de eyección, cociente colesterol total/HDL, índices de masa y de volumen de ventrículo izquierdo. El sexo, el hábito de fumar, la diabetes y la hipertensión arterial tampoco modificaron las diferencias entre grupos (datos no expuestos).

\section{DISCUSIÓN}

\section{RFC en insuficiencia cardíaca}

El presente estudio muestra por primera vez con PET que los pacientes en insuficiencia cardíaca de origen no isquémico, cualquiera que sea su etiología, tienen una RFC deprimida, que se relaciona con la clase funcional. La RFC fue 1,46 $\pm 0,48$ en pacientes en clase III-IV; $1,73 \pm 0,72$ y $1,89 \pm 0,75$ en pacientes en clase II (sin y con carvedilol, respectivamente) y $2,40 \pm 0,95$ en pacientes en clase I. Estas diferencias no son atribuibles a la edad, que fue similar en los cuatro grupos. Actualmente no está claro si la RFC disminuye en los ancianos (> 69 años) por deficiente dilatación de la microvasculatura coronaria ${ }^{20} \mathrm{o}$ por aumento del trabajo cardíaco (doble producto), que eleva el $\mathrm{FM}_{\mathrm{b}}{ }^{20}$. La RFC medida en voluntarios sanos de edad similar a la de nuestros pacientes ha sido 3,01 $\pm 0,73^{21}$. En nuestro estudio la diferencia de RFC entre pacientes de los grupos 1 y 4 fue significativa. Teóricamente la disfunción ventricular puede influir en la disminución de la RFC. Sin embargo, en nuestros pacientes, ni la fracción de eyección ni los índices de volumen ventricular, tomados como covariables, modificaron significativamente los resultados. Probablemente la clase funcional fue más determinante de la RFC que la enfermedad cardíaca subyacente.

\section{Estudios previos}

Estudios con PET y eco-Doppler intracoronario ${ }^{22}$ en pacientes con miocardiopatía dilatada e insuficiencia cardíaca han demostrado reducción de la RFC. El Doppler intracoronario muestra disfunción endotelial en pacientes con esta enfermedad, tanto microvascular como epicárdica ${ }^{23}$. La resistencia periférica está elevada en la insuficiencia cardíaca ${ }^{12}$ y el flujo hiperémico braquial está reducido, por disfunción endotelial ${ }^{13}$.

El estudio actual ha sido efectuado con ATP. Este fármaco, similar a la adenosina ${ }^{24}$, ha resultado útil en estudios de perfusión miocárdica ${ }^{25}$ y algunos ${ }^{26}$ han demostrado que su efecto vasodilatador es dependiente del endotelio. 


\section{Hipertrofia cardíaca}

La mayoría de nuestros pacientes tenía miocardiopatía dilatada o valvulopatía mitroaórtica. La miocardiopatía dilatada se asocia a una RFC reducida en ausencia de insuficiencia cardíaca, probablemente por anomalías de la vasodilatación ${ }^{7}$. La progresión de la enfermedad se asocia a mayor depresión de la perfusión miocárdica, tanto en reposo ${ }^{18}$ como tras dipirida$\mathrm{mol}^{7,18}$. Esta depresión no puede justificarse totalmente por la elevación de la presión diastólica de ventrículo izquierdo ni por la presión de perfusión coronaria reducida $^{27}$. La hipertensión arterial es otra causa de reducción de la $\mathrm{RFC}^{4}$, en parte debido a alteraciones arteriales estructurales. Sin embargo, está menos estudiada la RFC en la hipertrofia secundaria a valvulopatía. Un estudio ${ }^{8}$ muestra menor alteración de la RFC en la hipertrofia ventricular secundaria a estenosis aórtica que a hipertensión arterial. Nuestros pacientes tuvieron una disminución de la RFC relacionada con la clase funcional de insuficiencia cardíaca, y no con el tipo de cardiopatía subyacente. Estos hallazgos sugieren que la insuficiencia cardíaca, por sí misma, puede alterar la dilatación microvascular coronaria, independientemente de su causa. Esta alteración se añade a la producida por la cardiopatía de base.

\section{Factores neurohumorales}

En la insuficiencia cardíaca aumentan diversos factores neurohumorales e inflamatorios ${ }^{28-31}$ que pueden inducir vasoconstricción no sólo periférica, sino también coronaria. Es posible que disminuya la vasoconstricción cuando se controla la insuficiencia cardíaca y se reducen estos factores. Pensamos que ésta es la explicación más probable de nuestros hallazgos, aunque el estudio actual no permite confirmarlo. Tampoco se sabe cuánto tiempo es necesario para que se normalice la RFC cuando mejora clínicamente la insuficiencia cardíaca. Los pacientes del grupo 4, en grado funcional I, son los que tuvieron la RFC más alta. Aunque su fracción de eyección se había normalizado cuando se hizo el estudio, esta variable no tuvo relación significativa con la RFC. Este hecho puede indicar que la disminución de la RFC no se debe a la disminución de la necesidad de oxígeno ante una fracción de eyección reducida, sino a un problema de la microcirculación.

\section{Carvedilol}

Los pacientes del grupo 3, tratados con carvedilol, tuvieron tendencia a una RFC más alta que los del grupo 2, que estaban en la misma clase funcional, si bien la fracción de eyección fue algo más alta (sin significación estadística) en el grupo 3. Esto podría atribuirse al $\mathrm{FM}_{\mathrm{b}}$ reducido en el grupo 3 (tabla 3). Sin embargo, los pacientes del grupo 1 tuvieron RFC más baja (sin significación estadística) que los del grupo 3, a pesar de tener un $\mathrm{FM}_{\mathrm{b}}$ inferior (no significativa). El paciente estudiado en dos ocasiones distintas (con y sin carvedilol) tuvo un aumento de la RFC con carvedilol desproporcionado al aumento de la fracción de eyección. El carvedilol es un antagonista beta y alfa-1 adrenérgico, que además tiene propiedades antioxidantes ${ }^{32} \mathrm{y}$ antiendotelina- $1^{33}$. Estas propiedades pueden explicar, al menos en parte, la RFC ligeramente más alta en el grupo 3. Un estudio previo ${ }^{14}$ muestra que las modificaciones plasmáticas de la endotelina-1 reflejan la respuesta clínica al carvedilol en pacientes con insuficiencia cardíaca. Varios de los pacientes del grupo 4, que son los que tuvieron RFC más alta, también recibieron carvedilol.

\section{Limitaciones}

1. No se dispone de un quinto grupo de voluntarios sanos de edades similares para comparar con los cuatro grupos de pacientes, pero pensamos que se puede compararlos con los voluntarios sanos de edad similar de Czernin et $\mathrm{al}^{21}$, ya que estos autores utilizan un método similar al nuestro y obtienen valores similares a los nuestros (datos no publicados) en voluntarios jóvenes.

2. Lo ideal habría sido estudiar a los mismos pacientes en cuatro situaciones distintas durante su evolución, pero esto no resultó posible. La mayoría de los del grupo 1 recibió trasplante o falleció. Por otra parte, los pacientes del grupo 2 tenían alguna contraindicación para el tratamiento con bloqueadores beta.

3. El hecho de mezclar valvulopatías con miocardiopatías pudiera considerarse una limitación. Sin embargo, en las dos patologías se altera la RFC. En la figura 1 se observa que no hay diferencia apreciable en la desviación típica entre grupos; por tanto, desde el punto de vista estadístico no hay heterogeneidad entre ellos. Sólo hay diferencia significativa en la fracción de eyección, más alta en el grupo 4 , ya que fue criterio de selección para este grupo. Si el grupo 4 estuviese formado por voluntarios sanos, se debería atribuir su mayor RFC a la ausencia de cardiopatía. Sin embargo, está formado por cardiópatas cuya insuficiencia cardíaca ha mejorado; por tanto, se debe atribuir la diferencia con los restantes grupos al distinto grado funcional, una vez descartada la presencia de correlación entre variables hemodinámicas y RFC.

\section{IMPLICACIONES CLÍNICAS}

La RFC medida con PET aporta un mayor conocimiento de la fisiopatología microvascular coronaria en la insuficiencia cardíaca no isquémica. Los pacientes que están en mejor clase funcional son los que presentan niveles más altos de RFC. Si la baja RFC se debe a un problema microcirculatorio primario, como parece 
ser, la microcirculación debería ser un objetivo terapéutico. Son necesarios otros estudios con seguimiento evolutivo para establecer una posible relación entre RFC, factores neurohumorales e inflamatorios y pronóstico de los pacientes en insuficiencia cardíaca.

\section{AGRADECIMIENTOS}

Los autores agradecen a Iván Peñuelas la preparación de los radiotrazadores durante los estudios PET y a Javier Diez su asesoramiento científico.

\section{BIBLIOGRAFÍA}

1. Uren NG, Melin JA, De Bruyne B, Wijns W, Baudhuin T, Camici PG. Relation between myocardial blood flow and the severity of coronary artery stenosis. N Engl J Med 1994;330:1782-8.

2. Baller D, Notohamiprodjo G, Gleichmann U, Holzinger J, Weise $\mathrm{R}$, Lehmann J. Improvement in coronary flow reserve determined by positron emission tomography after 6 months of cholesterollowering therapy in patients with early stages of coronary atherosclerosis. Circulation 1999;99:2871-5.

3. Pitkanen OP, Nuutila P, Raitakari OT, Porkka K, Iida H, Nuotio I, et al. Coronary flow reserve is reduced in young men with IDDM. Diabetes 1998;47:248-54.

4. Gimelli A, Schneider-Eicke J, Neglia D, Sambuceti G, Giorgetti A, Bigalli G, et al. Homogeneously reduced versus regionally impaired myocardial blood flow in hypertensive patients: two different patterns of myocardial perfusion associated with degree of hypertrophy. J Am Coll Cardiol 1998;31:366-73.

5. Choudhury L, Elliott P, Rimoldi O, Ryan M, Lammertsma AA, Boyd H, et al. Transmural myocardial blood flow distribution in hypertrophic cardiomyopathy and effect of treatment. Basic Res Cardiol 1999;94:49-59.

6. Drzezga AE, Blasini R, Ziegler SI, Bengel FM, Picker W, Schwaiger M, et al. Coronary microvascular reactivity to sympathetic stimulation in patients with idiopathic dilated cardiomyopathy. J Nucl Med 2000;41:837-44.

7. Neglia D, Parodi O, Gallopin M, Sambuceti G, Giorgetti A, Pratali L, et al. Myocardial blood flow response to pacing tachycardia and to dipyridamole infusion in patients with dilated cardiomyopathy without overt heart failure. Circulation 1995;92:796-804.

8. Choudhury L, Rosen SD, Patel DP, Nihoyannopoulos P, Camici PG. Coronary flow reserve in primary and secondary left ventricular hypertrophy: a study with positron emission tomography. Eur Heart J 1997;18:108-16.

9. Knecht M, Burkhoff D, Yi GH, Popilskis S, Homma S, Packer $\mathrm{M}$, et al. Coronary endothelial dysfunction precedes heart failure and reduction of coronary reserve in awake dogs. J Mol Cell Cardiol 1997;29:217-27.

10. Drexler H, Hornig B. Importance of endothelial function in chronic heart failure. J Cardiovasc Pharmacol 1996;26(Suppl 2):S9-12.

11. Dexler H, Hornig B. Endothelial dysfunction in human disease. J Mol Cell Cardiol 1999;31:51-60.

12. Katz SD. The role of endothelium-derived vasoactive substances in the pathophysiology of exercise intolerance in patients with congestive heart failure. Prog Cardiovasc Dis 1995;38:23-50.

13. Takeshita A, Hirooka Y, Imaizumi T. Role of endothelium in control of forearm blood flow in patients with heart failure. $\mathrm{J}$ Card Fail 1996;2:S209-15.

14. Krum H, Gu A, Wilshire-Clement M, Sackner-Bernstein J,
Goldsmith R, Medina N, et al. Changes in plasma endothelin-1 levels refects clinical response to b-blockade in chronic heart failure. Am Heart J 1996;131:337-41.

15. Camici PG. Positron emission tomography and myocardial imaging. Heart 2000;82:475-80.

16. Agustí Escasany A, Durán Dalmau M, Arnau de Bolós JM, Rodríguez Cumplido D, Diogène Fadini E, Casas Rodríguez J, et al. Tratamiento médico de la insuficiencia cardíaca basado en la evidencia. Rev Esp Cardiol 2001;54:715-34.

17. Muzik O, Beanlands R, Wolfe E, Schwaiger M. Automated region definition for cardiac nitrogen-13-ammonia PET imaging. J Nucl Med 1993;34:336-44.

18. Muzik O, Beanlands RSB, Hutchins GD, Mangner TJ, Nguyen N, Schwaiger M. Validation of nitrogen-13-ammonia tracer kinetic model for quantification of myocardial blood flow using PET. J Nucl Med 1993;34:83-91.

19. Shikama N, Himi T, Yoshida K, Nakao M, Fujiwara M, Tamura $\mathrm{T}$, et al. Prognostic utility of myocardial blood flow assessed by $\mathrm{N}-13$ ammonia positron emission tomography in patients with idiopathic dilated cardiomyopathy. Am J Cardiol 1999;84:434-9.

20. Uren NG, Camici PG, Melin JA, Bol A, de Bruyne B, Radvan J, et al. Effect of aging on myocardial perfusion reserve. J Nucl Med 1995;36:2032-6.

21. Czernin J, Muller P, Chan S, Brunken RC, Porenta G, Krivokapich J, et al. Influence of age and hemodynamics on myocardial blood flow and flow reserve. Circulation 1993;88:62-9.

22. Merlet P, Mazoyer B, Hittinger L, Valette H, Saal JP, Bendriem $\mathrm{B}$, et al. Assessment of coronary reserve in man: comparison between positron emission tomography with oxygen-15-labeled water and intracoronary Doppler technique. J Nucl Med 1993;34: 1899-904.

23. Mathier MA, Rose GA, Fifer MA, Miyamoto MI, Dinsmore RE, Castano $\mathrm{HH}$, et al. Coronary endothelial dysfunction in patients with acute-onset idiopathic dilated cardiomyopathy. J Am Coll Cardiol 1998;32:216-24.

24. Jeremias A, Filardo SD, Whitbourn RJ, Kernoff RS, Yeung AC, Fitzgerald PJ, et al. Effects of intravenous and intracoronary adenosine 5'-triphosphate as compared with adenosine on coronary flow and pressure dynamics. Circulation 2000;101:318-23.

25. Bravo N, Giménez M, Mejía S, García-Velloso MJ, ComaCanella I. Prognostic value of myocardial perfusion imaging with adenosine triphosphate. J Nuclear Cardiol 2002;9:395-401.

26. De Mey JG, Vanhoutte PM. Role of the intima in cholinergic and purinergic relaxation of isolated canine femoral arteries. J Physiol (Lond) 1981;316:347-55.

27. Nitenberg A, Foult JM, Blanchet F, Zouioueche S. Multifactorial determinants of reduced coronary flow reserve after dipyridamole in dilated cardiomyopathy. Am J Cardiol 1985;55:748-54.

28. Tsutamoto T, Wada A, Maeda K, Hisanaga T, Mabuchi N, Hayashi M, et al. Plasma brain natriuretic peptide level as a biochemical marker of morbidity and mortality in patients with asymptomatic or minimally symptomatic left ventricular dysfunction. Comparison with plasma angiotensin II and endothelin-1. Eur Heart J 1999;20:1799-807.

29. Adamopoulos S, Parissis J, Kroupis C, Kroupis C, Georgiadis M, Karavolias G, et al. Physical training reduces peripheral markers of inflammation in patients with chronic heart failure. Eur Heart $\mathbf{J}$ 2001;22:791-7.

30. Vidal B, Roig E, Pérez-Villa F, Orús J, Pérez J, Jiménez V, et al. Valor pronóstico de los niveles de citocinas y neurohormonas en la insuficiencia cardíaca severa. Rev Esp Cardiol 2002;55:481-6.

31. Herrera Garza EH, Herrera Garza JL, Rodríguez González H, Treviño A, Ibarra Flores M, Torre Amione G. Importancia del factor de necrosis tumoral alfa en la patogenia de la insuficiencia cardíaca. Rev Esp Cardiol 2002;55:61-6.

32. Feuerstein GZ, Bril A, Ruffolo RR Jr. Protective effects of carvedilol in the myocardium. Am J Cardiol 1997;80(Suppl):L41-5.

33. Ohlstein EH, Arleth AJ, Storer B, Romanic AM. Carvedilol inhibits endothelin-1 biosynthesis in cultured human coronary artery endothelial cells. J Mol Cell Cardiol 1998;30:167-73. 